\title{
EDITORIAL
}

\section{Síndrome del Intestino Irritable: lo (pen)último sobre lo que se está investigando.}

Juan J. Sebastián Domingo

Consulta de Trastornos Funcionales Digestivos.

Servicio de Aparato Digestivo

Hospital Royo Villanova

Zaragoza

Sobre el Síndrome del Intestino Irritable (SII) todos los licenciados o graduados en Medicina, y los propios pacientes, hemos leído, escuchado y comentado algunos aspectos, información más o menos reciente y contrastada, procedente de libros, artículos de revista, cursos, conferencias, Internet, etc., que no se trata de repetir aquí. Lo que pretende esta Editorial es repasar y hacer llegar a los lectores de Medicina Clínica los últimos avances científicos, las últimas evidencias, que se están publicando sobre este trastorno, tan frecuente como poco (o no demasiado) conocido todavía. Para empezar, si bien el SII desde siempre se ha entendido como un trastorno funcional, y así está clasificado en los Criterios Roma III -grupo C1(http://romecriteria.org/assets/pdf/19_RomeIII_apA_885-898.pdf), cada vez hay más evidencias que apuntan a que esto no parece estar tan claro, puesto que se están detectando alteraciones orgánicas sutiles, muchas de ellas a nivel celular, bioquímico (neurotransmisores, mediadores inmunitarios, citoquinas) o microbiológico (microorganismos difíciles de descubrir o cultivar o, incluso, desconocidos o no bien tipificados), como ya apuntaba en una Editorial en esta misma revista (1). Por otro lado, en cuanto a la etiopatogenia, nuestro grupo de trabajo ya apuntó, hace más de 6 años, el probable origen inmune del trastorno (2,3), que también se ha considerado en revisiones más recientes sobre el tema $(4,5)$, y sobre el que hemos seguido investigando. Véase, en este sentido, el posible papel de la Psiconeuroinmunología en el mismo (6), proponiendo un modelo que podría explicar la 
fisiopatología en el tipo SII-postinfeccioso. En dicho modelo, la activación inmune intestinal alterada, la permeabilidad intestinal, y el microbioma intestinal y colónico parecen jugar un papel relevante (7).

En los últimos años, parece que se está avanzando en encontrar algún biomarcador en el SII. Se ha propuesto que la densidad celular de cromogranina A duodenal, que es un marcador común de células endocrinas, se puede utilizar como un biomarcador para el diagnóstico de SII, con una sensibilidad y especificidad de 91 y $89 \%$, respectivamente (8). El Péptido YY rectal (PYY) puede considerarse otro posible biomarcador, con una sensibilidad y especificidad del 83 y 89\% (9). La densidad de ambos tipos de células está sensiblemente reducida en los pacientes con SII.

Otro potencial biomarcador es la calprotectina fecal $(C F)$, que puede utilizarse en particular para hacer el diagnóstico diferencial con la enfermedad inflamatoria intestinal (EII): la CF se confirma como un biomarcador altamente específico y sensible para la EII y un valor de $50 \mathrm{mcg} / \mathrm{g}$ muestra una sensibilidad del $93 \%$ y $94 \%$ de especificidad en la diferenciación de la EII del SII (10).

Finalmente, otro marcador fecal de valor potencial es la $\beta$-defensin-2 humana (HBD-2). Se trata de un péptido que es producido por la mucosa del colon inflamado, y que apenas existe en el colon no inflamado. Su producción depende de la actividad de los microorganismos y citoquinas proinflamatorias. Langhorst et al. (11) han encontrado niveles de HBD-2 significativamente más altos en pacientes con SII en comparación con controles sanos.

Una buena y reciente revisión acerca del papel de los potenciales biomarcadores en el SII puede encontrarse en el estudio publicado por Ivana Plavši 'c et al. (12) En los últimos años, cada vez se le está dando más importancia a la dieta en este trastorno. Las intolerancias alimentarias son comunes en pacientes con SII. Cada vez 
más, los hidratos de carbono de cadena corta, osmóticamente activos y rápidamente fermentables (incluyendo fructosa, lactosa, fructanos y galactanos, y alcoholes del azúcar) han sido reconocidos como un disparador importante de los síntomas del SII. Los carbohidratos mal absorbidos pueden ejercer efectos osmóticos y dar lugar a un aumento de la fermentación en el intestino delgado o el colon, lo que puede exacerbar los síntomas en estos pacientes, que ya tienen anormalidades subyacentes en la función y la sensibilidad intestinales (13). Este tipo de dieta se conoce como FODMAP (fermentable oligosaccharides, disaccharides, monosaccharides, and polyols). El tratamiento del SII sigue siendo sintomático y tangencial, en el sentido de que no puede enfrentarse a una causa concreta o bien establecida. Los resultados de cualquier terapia empleada en el SII siguen siendo más bien moderados y, en cualquier caso, transitorios. Se están investigando diferentes tratamientos que abordan alguno de los múltiples mecanismos fisiopatológicos que parecen estar detrás del síndrome. Tratando de sistematizarlos, me voy a referir, de forma sucinta, a las últimas revisiones sobre las opciones terapéuticas más novedosas empleadas en estos momentos.

1. Papel de la dieta: La manipulación dietética en los pacientes con SII pretende identificar ingredientes alimentarios que potencialmente puedan desencadenar síntomas intestinales, tales como fructosa, lactosa, fructanos o sorbitol (FODMAP), y eliminarlos de forma selectiva de la dieta, lo que puede ocasionar un alivio (parcial y transitorio) de los síntomas en estos pacientes y restaurar la función normal del intestino. Este tipo de dieta está sistematizada en tablas para facilitar y mejorar su empleo por parte de los pacientes (ver en http://www.dolor-abdominal.com/nutrisiia/fodmaps.html). Una revisión sistemática reciente sobre el empleo de dietas restringidas en FODMAP en el manejo del SII (y estreñimiento crónico) concluye que, aunque este tipo de dieta puede ser eficaz en el tratamiento a corto plazo de pacientes seleccionados con SII (nivel de 
evidencia II; grado de recomendación C), se precisa de ensayos clínicos aleatorizados más rigurosos y a largo plazo para establecer la eficacia y seguridad de estas dietas restrictivas, particularmente sobre la salud del colon y el microbioma a largo plazo (14).

2. Linaclotida: La linaclotida es un agonista de los receptores de la guanilato ciclasa tipo C aprobado, en septiembre de 2012, por la Agencia Europea del Medicamento (EMA) para el tratamiento sintomático de adultos con SII moderado a grave con estreñimiento que no han respondido a otras medidas menos específicas (ver en http://tinyurl.com/nmt9yuv). En diferentes estudios, ha demostrado su perfil de eficacia y seguridad $(15,16)$. En un reciente meta-análisis, se constató un alivio significativo del estreñimiento y la calidad de vida en el $45 \%$ de los pacientes tratados con linaclotida, frente al 23,3\% del grupo placebo (15). Por tanto, linaclotida parece moderadamente eficaz para mejorar los síntomas del SII con estreñimiento.

3. Rifaximina: La rifaximina es un antibiótico de amplio espectro, de uso oral, no sistémico, que tiene su diana en el intestino y se asocia con un bajo riesgo de resistencia bacteriana. Se ha mostrado eficaz en el manejo del SII sin estreñimiento, proporcionando un alivio significativo de los síntomas, tanto de la hinchazón, como del dolor abdominal y las deposiciones blandas o acuosas $(17,18)$. En un reciente análisis combinado de varios ensayos clínicos randomizados, doble ciego y controlados con placebo (19), parece que el perfil de seguridad y tolerabilidad de rifaximina durante y tras el tratamiento en pacientes con SII sin estreñimiento fue comparable al del placebo. 4. Trasplante de microbiota fecal: Brandt et al (20), en una reciente e interesante revisión acerca de las indicaciones del trasplante de microbiota fecal (TMF), apuntan a que ha sido utilizada con éxito en pacientes con SII, al igual que otros autores $(21,22)$, encontrando mejoría o resolución de los síntomas en el 70\% de los pacientes, tanto del 
dolor (72\%) como de la distensión abdominales (50\%). A largo plazo, el 46\% de los pacientes experimentó un aumento significativo de su bienestar general.

5. Probióticos: Una reciente revisión sistemática y meta-análisis sobre la eficacia de los probióticos en el SII, parece indicar que son tratamientos efectivos, si bien es poco probable que la mayoría de las terapias con probióticos sean beneficiosas para todos los pacientes, y que deben utilizarse especies y cepas individuales de forma más o menos específica para cada tipo de paciente y de síntoma predominante (23).

6. Melatonina: La melatonina juega un papel importante en la fisiología gastrointestinal, incluyendo la regulación de la motilidad, acción local antiinflamatoria y antinociceptiva, así como en la moderación de la sensibilidad visceral. En este sentido, los estudios llevados a cabo con melatonina (3 mg.) en el tratamiento del SII han demostrado, de forma consistente, una mejoría del dolor abdominal y de la calidad de vida en el 43,63\% en el grupo de la melatonina frente al $14,64 \%$ en el grupo placebo en pacientes con SII (24), aparte, claro está, de la promoción del sueño en estos pacientes.

7. Antidepresivos: Tanto los antidepresivos tricíclicos (ADT) como los inhibidores selectivos de la recaptación de la serotonina (ISRS) han demostrado su eficacia en el tratamiento de los pacientes con SII (25). En cuanto a los primeros, el 43,3\% de los pacientes tratados con ADT no obtuvieron mejoría de los síntomas, frente al 63,7\% en los sujetos que recibieron placebo. Y, respecto a los ISRS, el 45,5\% de los pacientes tratados con estos fármacos no tuvo ninguna mejoría de los síntomas tras el tratamiento, frente al $67,2 \%$ de los sujetos tratados con el placebo (25). Sigue siendo controvertido si el beneficio de los antidepresivos en estos pacientes depende del tratamiento de la depresión, que coexiste en muchos de esos casos, o de un efecto primario sobre el propio SII y sus mecanismos. 
8. Terapias psicológicas: Mindfulness y ACT. La reducción del estrés basada en Mindfulness (REBM; MBSR, en inglés) es un grupo de intervenciones psicosociales consistente en la práctica de meditación tipo mindfulness (atención plena) y estiramientos suaves de yoga hatha que se han estado aplicando dentro de algunas poblaciones con enfermedades crónicas, con el objetivo de reducir el estrés y los síntomas de la enfermedad (26).

En uno de los estudios de referencia sobre este tipo de intervención (26), se confirmó que la gravedad de los síntomas del SII mejoró de forma muy significativa $(\mathrm{p}<0.0001)$ antes y después de la intervención en el grupo REBM (30,7\% de reducción de los síntomas en ese grupo frente a 5,2\% en los controles), y los resultados se mantuvieron a los 6 meses de seguimiento.

Por otro lado, la Terapia de Aceptación y Compromiso (ACT) es un nuevo enfoque de intervención, dentro de las terapias conductuales llamadas de tercera generación, en el que no se pretende reducir los síntomas sino construir la habilidad de convivir con ellos, llevando una vida lo más normal posible (27). Una intervención ACT puede mejorar la gravedad de los síntomas y la calidad de vida del paciente con SII, debido a la reducción de los comportamientos de evitación y al aumentar la flexibilidad psicológica del paciente (27). Se puede seguir viviendo pese a tener un intestino irritable.

9. Medicinas Complementarias: En un meta-análisis de 6 ECA sobre la eficacia de la acupuntura en el tratamiento del SII, cinco artículos no mostraron ningún beneficio de aquella en el tratamiento del proceso, y solo uno sugirió una mejoría de los síntomas del SII, incluyendo dolor y distensión abdominal, sensación de evacuación incompleta, número de deposiciones por día y características de las heces. En dicha mejoría parece estar implicada la serotonina (28). En cuanto a la homeopatía, una Revisión Sistemática de la Colaboración Cochrane (29) refiere que no pueden extraerse conclusiones debido a 
la baja calidad de los ensayos clínicos realizados y analizados (solamente dos), a posibles sesgos en la realización de los mismos y al corto período de seguimiento de los pacientes. Finalmente, en cuanto al tratamiento con hierbas de uso medicinal, en un solo ECA multicéntrico, doble ciego y controlado con placebo, los pacientes tratados con el preparado herbal STW5 (Iberogast), compuesto de extractos de nueve plantas medicinales, experimentaron una reducción significativa en las puntuaciones de dolor abdominal durante el tratamiento con dicho preparado $(\mathrm{p}=0.009)$ comparado con el placebo después de un tratamiento durante cuatro semanas (30).

Para terminar, sugiero que se cambie la abstracción taquigráfica de Síndrome del Intestino Irritable, por la de Síndrome de Intestino Sensible, a mi juicio más apropiada, a tenor de lo (todavía poco) que conocemos actualmente sobre el mismo.

A modo de conclusión, cabría añadir que, pese a todo lo que se sigue investigando sobre este trastorno funcional digestivo, es más bien poco lo que se está avanzando en el tratamiento efectivo de los síntomas, que es lo que interesa a los pacientes. Todavía, en estos momentos, el tratamiento no parece estar actuando de forma esencial sobre la etiopatogenia del proceso. 


\section{BIBLIOGRAFÍA}

1. Sebastián Domingo JJ. The irritable bowel syndrome, should not be considered a functional disorder? Med Clin (Barc). 2013;140(9):403-5.

2. Ortiz-Lucas M, Saz-Peiró P, Sebastián-Domingo JJ. Irritable bowel syndrome immune hypothesis. Part one: the role of lymphocytes and mast cells. Rev Esp Enferm Dig. 2010;102(11):637-47.

3. Ortiz-Lucas M, Saz-Peiró P, Sebastián-Domingo JJ. Irritable bowel syndrome immune hypothesis. Part two: the role of cytokines. Rev Esp Enferm Dig. 2010;102(12):711-7.

4. El-Salhy M, Hatlebakk JG, Gilja OH, Hausken T. Irritable bowel syndrome: recent developments in diagnosis, pathophysiology, and treatment. Expert Rev Gastroenterol Hepatol. 2014;8(4):435-43.

5. Shulman RJ, Jarrett ME, Cain KC, Broussard EK, Heitkemper MM. Associations among gut permeability, inflammatory markers, and symptoms in patients with irritable bowel syndrome. J Gastroenterol. 2014;49(11):1467-76.

6. Sebastián Domingo JJ, Sebastián Sánchez B. Psychoneuroimmunology in irritable bowel síndrome. Gastroenterol Hepatol. 2015;38(7):413-6.

7. Dupont HL. Review article: evidence for the role of gut microbiota in irritable bowel syndrome and its potential influence on therapeutic targets. Aliment Pharmacol Ther. 2014;39(10):1033-1042.

8. El-Salhy M, Lomholt-Beck B, Hausken T. Chromogranin A as a possible tool in the diagnosis of irritable bowel syndrome. Scand J Gastroenterol 2010;45:1435-9.

9. El-Salhy M, Gundersen D, Hatlebakk JG, Hausken T. Abnormal rectal endocrine cells in patients with irritable bowel syndrome. Regul Pept 2014;188:60-5.

10. Waugh N, Cummins E, Royle P, Kandala NB, Shyangdan D, Arasaradnam R, Clar C, Johnston R. "Faecal calprotectin testing for differentiating amongst inflammatory and noninflammatory bowel diseases: systematic review and economic evaluation. Health Technol Assess 2013; 17 (55)

11. J. Langhorst, A. Junge, A. Rueffer et al., "Elevated human $\beta$-defensin-2 levels indicate an activation of the innate immune system in patients with irritable bowel syndrome,” Am J Gastroenterol. 2009 ;104(2):404-10.

12. Plavšić I, Hauser G, Tkalčić M, Pletikosić S, Salkić N. Diagnosis of Irritable Bowel Syndrome: Role of Potential Biomarkers. Gastroenterol Res Pract. 2015;2015: 490183

13. Farre R, Tack J. Food and symptom generation in functional gastrointestinal disorders: physiological aspects. Am J Gastroenterol. 2013;108 (5):698-706. 
14. Rao SS, Yu S, Fedewa A. Systematic review: dietary fibre and FODMAP-restricted diet in the management of constipation and irritable bowel syndrome. Aliment

Pharmacol Ther. 2015;41(12):1256-70.

15. Atluri DK, Chandar AK, Bharucha AE, Falck-Ytter Y. Effect of linaclotide in irritable bowel syndrome with constipation (IBS-C): a systematic review and metaanalysis. Neurogastroenterol Motil. 2014;26(4):499-509.

16. Carballo F. Linaclotide in the treatment of patients with irritable bowel syndrome and constipation - analysis of an opportunity. Rev Esp Enferm Dig. 2013;105(6):34554.

17. Pimentel M, Lembo A, Chey WD, Zakko S, Ringel Y, Yu J, Mareya SM, Shaw AL, Bortey E, Forbes WP; TARGET Study Group. Rifaximin therapy for patients with irritable bowel syndrome without constipation. N Engl J Med. 2011;364(1):22-32.

18. Brandt LJ, Chey WD, Foxx-Orenstein AE, et al. An evidence-based position statement on the management of irritable bowel syndrome. Am J Gastroenterol 2009; 104(Suppl. 1): S1-35.

19. Schoenfeld P, Pimentel M, Chang L, Lembo A, Chey WD, Yu J, Paterson C, Bortey E, Forbes WP. Safety and tolerability of rifaximin for the treatment of irritable bowel syndrome without constipation: a pooled analysis of randomised, double-blind, placebocontrolled trials. Aliment Pharmacol Ther. 2014;39(10):1161-8.

20. Brandt LJ, Aroniadis OC. An overview of fecal microbiota transplantation: techniques, indications, and outcomes. Gastrointest Endosc. 2013;78(2):240-9.

21. Pinn DM, Aroniadis OC, Brandt LJ. Is fecal microbiota transplantation the answer for irritable bowel syndrome? A single-center experience. Am J Gastroenterol. 2014; 109(11):1831-2.

22. Foxx-Orenstein AE, Chey W. Manipulation of the gut microbiota as a novel treatment strategy for gastrointestinal disorders. Am J Gastroenterol Suppl 2012;1:41-6.

23. Ford AC, Quigley EM, Lacy BE, Lembo AJ, Saito YA, Schiller LR, Soffer EE, Spiegel BM, Moayyedi P. Efficacy of prebiotics, probiotics, and synbiotics in irritable bowel syndrome and chronic idiopathic constipation: systematic review and metaanalysis. Am J Gastroenterol. 2014;109(10):1547-61.

24. Siah KT, Wong RK, Ho KY. Melatonin for the treatment of irritable bowel syndrome. World J Gastroenterol. 2014;20(10):2492-8.

25. Ford AC, Quigley EM, Lacy BE, Lembo AJ, Saito YA, Schiller LR, Soffer EE, Spiegel BM, Moayyedi P. Effect of antidepressants and psychological therapies, including hypnotherapy, in irritable bowel syndrome: systematic review and metaanalysis. Am J Gastroenterol. 2014;109(9):1350-65. 
26. Zernicke KA, Campbell TS, Blustein PK, Fung TS, Johnson JA, Bacon SL, Carlson LE. Mindfulness-based stress reduction for the treatment of irritable bowel syndrome symptoms: a randomized wait-list controlled trial. Int J Behav Med. 2013;20(3):385-96.

27. Ferreira NB, Eugenicos MP, Morris PG, Gillanders DT. Measuring acceptance in irritable bowel syndrome: preliminary validation of an adapted scale and construct utility. Qual Life Res. 2013;22(7):1761-6.

28. Chao GQ, Zhang S. Effectiveness of acupuncture to treat irritable bowel syndrome: a meta-analysis. World J Gastroenterol. 2014;20(7):1871-7.

29. Peckham EJ, Nelson EA, Greenhalgh J, Cooper K, Roberts ER, Agrawal A. Homeopathy for treatment of irritable bowel syndrome. Cochrane Database Syst Rev. 2013;11: CD009710.

30. Madisch A, Holtmann G, Plein K, Hotz J. Treatment of irritable bowel syndrome with herbal preparations: results of a double-blind, randomized, placebo-controlled, multi-centre trial. Aliment Pharmacol Ther. 2004;19(3):271-9. 